\title{
Rapid Adoption of Telehealth at an Interprofessional Student-Run Free Clinic
}

Ryan Cao Vinh Phan | Dung Van Le | Alexander Nguyen | Kari Mader, MD, MPH

PRiMER. 2020;4:23.

Published: 9/24/2020 | DOI: 10.22454/PRiMER.2020.241619

\section{Abstract}

Introduction: Student-run free clinics (SRFCs) have become important primary care homes during the COVID-19 pandemic. With students pulled from clinical sites, funding deficits, SRFCs' voluntary nature, and no best practices for telehealth SRFCs, many have been forced to close. This report shares a systematic approach for implementing a telehealth clinic along with initial outcomes from the Dedicated to Aurora's Wellness and Needs (DAWN) SRFC.

Methods: We utilized pilots with students, community volunteers, and patients to identify a telehealth platform. We implemented weekly plan-do-study-act (PDSA) cycles to develop a feasible interprofessional telehealth model. Key PDSA cycle goals included seamless utilization of platform, identification of necessary team members, appropriate scheduling of patients and volunteers, integration of interprofessional learners, positive patient and volunteer experience, and process for identifying and addressing patient social needs. Measured outcomes included total visits, no-show rates, and chief complaints addressed.

Results: Outcomes from PDSA cycles included a resultant telehealth clinic team and model, workflow for outreach for social needs screening and navigation, and team training guides. Visit data and no-show rates from January 2020 through July 2020 demonstrated total visits returned to $60 \%$ of pre-COVID numbers while no-show rates decreased significantly below pre-COVID rates. A range of acute and chronic concerns were successfully managed via telehealth.

Conclusion: SRFCs are poised to continue serving an important role in caring for the country's most vulnerable populations. The DAWN telehealth implementation process, outcomes, and resultant protocols may help inform other SRFCs seeking to establish telehealth services.

\section{Introduction}

Since March 2020, communities have adopted social distancing policies to limit COVID-19 cases. ${ }^{1}$ Medical schools nationwide withdrew students from clinical duties and transitioned to remote learning. The pandemic created disproportionate strain on many vulnerable populations by exacerbating inequities in health care access, wealth, and income volatility. ${ }^{2}$ Student-run free clinics (SRFCs), essential primary care homes to many underserved populations, are present at $75 \%$ of Association of American Medical Colleges (AAMC) member institutions with $74 \%$ of 2019 medical school graduates participating. ${ }^{3,4}$ SRFCs were forced to confront concerns of asymptomatic viral transmission and limited protective equipment.

The Dedicated to Aurora's Wellness and Needs (DAWN) interprofessional SRFC in Aurora, Colorado integrates licensed interprofessional providers with over 500 student volunteers per year from dental, medicine, nurse 
practitioner, nursing, physician assistant, pharmacy, physical therapy, prehealth, and psychology programs. Forty student volunteers weekly serve uninsured adults of all ages, $45 \%$ of whom are male and $55 \%$ female. Approximately $70 \%$ identify as Hispanic/Latino and $18 \%$ as Black/African American. Upon establishing care, $91 \%$ do not have a usual source of care and over $50 \%$ report their health as poor or fair.

Motivated to ensure patients still had services, DAWN reviewed the literature and found limited examples of how to implement a telehealth SRFC. ${ }^{5-12}$ In March 2020, displaced DAWN students initiated development of an interprofessional telehealth model and recorded the systematic approach and initial outcomes from implementation to support other SRFCs desiring to initiate telehealth.

\section{Methods}

This project met the definition of a quality improvement project, thus did not require review by the Colorado Institutional Review Board.

\section{Preparing for Implementation}

DAWN student leaders met weekly starting March 11, 2020 to develop an initial telehealth workflow based on literature review and the historical in-person model. Leaders identified and piloted six telehealth options, and then top platforms were piloted with volunteer community members and finally with patients over several clinic sessions before Zoom was selected. ${ }^{13}$ Telehealth options can be found in the STFM Resource Library. ${ }^{14}$

\section{Implementation}

We utilized a plan-do-study-act (PDSA) framework starting April 7, 2020 for implementing telehealth with goals including seamless utilization of platform, identification of necessary team members, appropriate scheduling of patients and volunteers, integration of interprofessional learners, positive patient and volunteer experience, and process for identifying and addressing patient social needs. We completed weekly PDSA cycles with DAWN Virtual Clinic leadership as shown in Figure 1. We incorporated staged changes according to key PDSA cycle goals over an 8-week period until the model reached relative stability. We obtained short-term outcome measures via manual chart review including total medical and clinical pharmacy visits by month, average no-show rates by month, frequency of chief complaints addressed via telehealth, and reasons for conversion from a telehealth visit to inperson follow-up, if applicable.

\section{Results}

We developed a student telehealth training guide and Zoom instruction guide based on the literature review along with consensus feedback from student leaders. The guides can be found in the STFM Resource Library. ${ }^{15,16}$ Virtual student medical and clinical pharmacy clinics with continuous PDSA-guided improvements resulted in a nearfinalized model by May 2020. See Figure 2 for the resultant volunteer roles, model, and clinic flow. Lastly, the goal to respond to increased patient social needs resulted in the creation of a workflow leveraging student leaders for nonclinical outreach related to social determinants of health (Figure 3).

Visit data and no-show rates from January 2020 through July 2020 are shown in Table 1. Total visits in June and July recovered to $60 \%$ capacity of pre-COVID visits in January and February. With the implementation of telehealth at DAWN, no-show rates in June and July decreased significantly from pre-COVID rates. Approximately three-quarters of total telehealth visits were completed via interpreters in six languages-Spanish, Twi, Tigrigna, Nepali, Korean, and Marshallese.

The range of chief complaints addressed via telehealth visits are noted in Table 2. Notably, it was possible for hypertension to be exclusively managed via telehealth if a patient had a home blood pressure cuff. Two acute concerns that required in-person follow-up $100 \%$ of the time were patients discharged from the hospital on oxygen and patients requiring a volume examination. 


\section{Conclusion}

To continue serving the community in the COVID-19 era, SRFCs need immediate telehealth solutions to develop effective models of care. DAWN's success with integrating telehealth is the result of the tireless work of a large group of clinic leaders over numerous hours. No formal data were gathered to assess patient or volunteer experience. Attendings, residents, and students provided positive feedback regarding clinical care during the pandemic as well as fluidity and rapid improvement of the telehealth model during weekly debriefs. For SRFCs seeking to develop their own telehealth model, it is imperative to have dedicated volunteers able to engage consistently over several months coupled with a standardized process for reflection and improvement.

A notable finding in this review was an improvement in no-show rates to better than pre-COVID rates by the end of the study period. This may reflect the accessibility of telehealth, warm handoffs between team members, or increased follow-up between care coordinators and patients within this new model. One related limitation was that the visit cancellations in the medical record may not have been fully updated in March and April 2020, making the apparent improvement from April through July more impressive than actuality. This effect was minimized through manual review of all visits. Lastly, this report is limited in that it pertains to only one clinic site, has limited short-term outcomes due to data extraction options, and is contextual to the local resources and prior clinic model.

DAWN's response to complex needs during this unprecedented era is a model with materials and lessons that can be instructive to other settings and communities. Beyond SRFCs, this example shares affordable telehealth solutions for any clinic serving similar populations, and demonstrates how to integrate interprofessional learners into a virtual care setting for the field of medical education.

\section{Tables and Figures}


Figure 1: PDSA Cycle Overview

\section{Weekly PDSA Timeline}

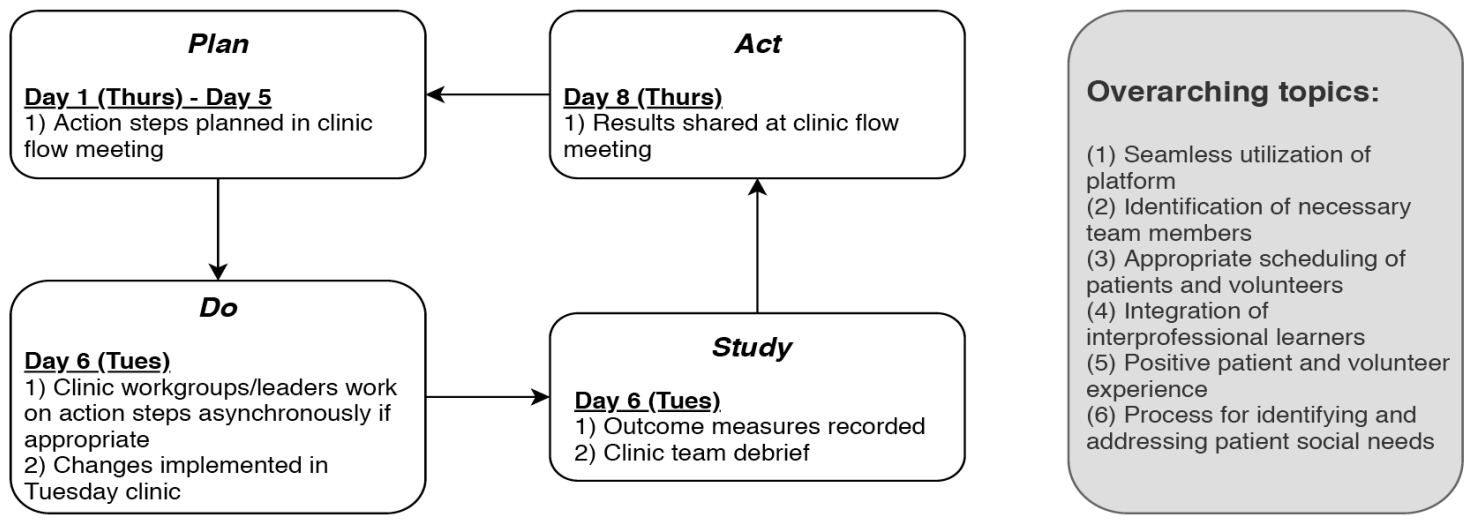

\section{DAWN Virtual Clinic Leadership}

1) Subspecialty Services

2) Diabetes Education

3) Behavioral Health

4) Physical Therapy

5) Dental

1) Community Partners

2) Community Engagement

3) Community Leaders of DAWN
1) $M D / P A / N P$

2) Pharmacy

3) Nursing

1) Interpreter

2) Registration

3) Clinic Managers

4) Care Coordination 
Figure 2: Clinical Care Workflow

Pre-Clinic Visit
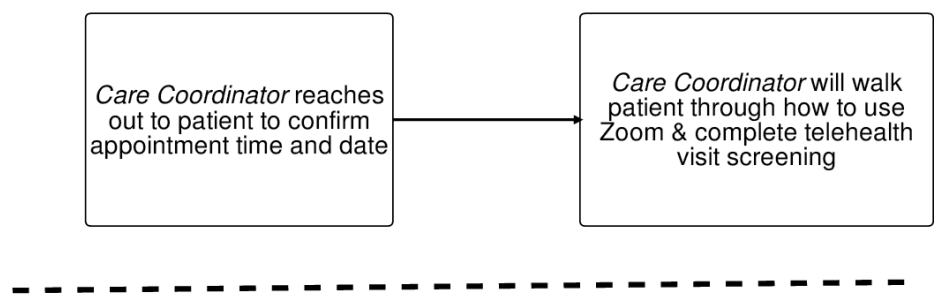

\section{Telemedicine}

Clinic Visit

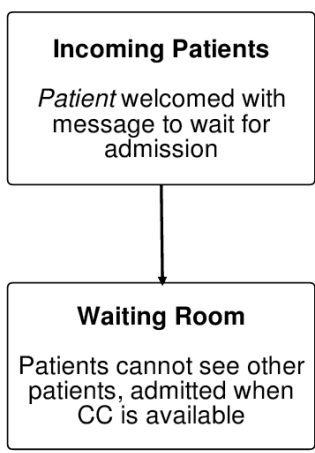

\section{Telemedicine Workflow Walkthrough}

\section{Telemedicine Team Members}

1. Student Interpreter, as needed

2. Student Care Coordinator - Pre-Health

3. Student Learner - MD/PA/NP/NS

4. Student Team Lead - Senior MD/PA/NP

5. Student Clinic Manager

6. Resident

7. Medicine Preceptor

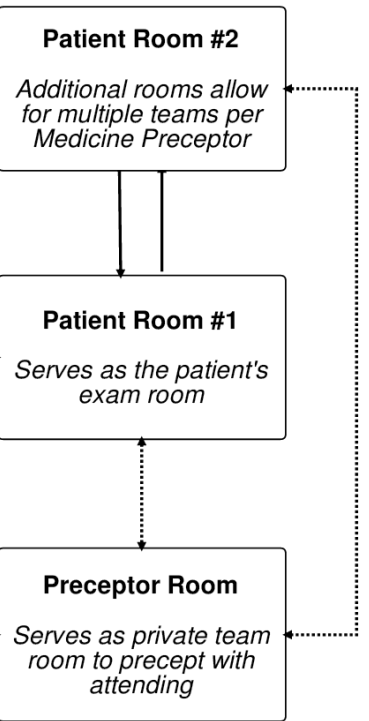

Part I: Pre-Clinic Team Huddle

- Review patients' charts for the night in Main Room

- Confirm team communication through Epic Secure Chat during patient visits Students' learning goals

Part II: Interview Patient ( 30 minutes)

- Team transitions to Patient Room obtains patient's verbal consent

- Student team introduces team members, sets expectations, and conducts the visit

- One student writes notes including assessment/plan in the EMR

- Resident or Preceptor observes visit

Part III: Preceptorship ( 15 minutes)

- Student Team presents abbreviated presentation to Resident or Preceptor in

Preceptor Room (or in Patient Room)

- Resident or Preceptor finalizes plan with the Student team

Part IV: Conclusion ( 10 minutes)

- Team returns to Patient $\boldsymbol{R o o m}$, discusses assessment and plan with the patient - Care Coordinator schedules any follow-up (Labs, telehealth/in-person visits, etc.) 
Figure 3: Asynchronous Care Workflow

\section{Holistic Care Outreach}

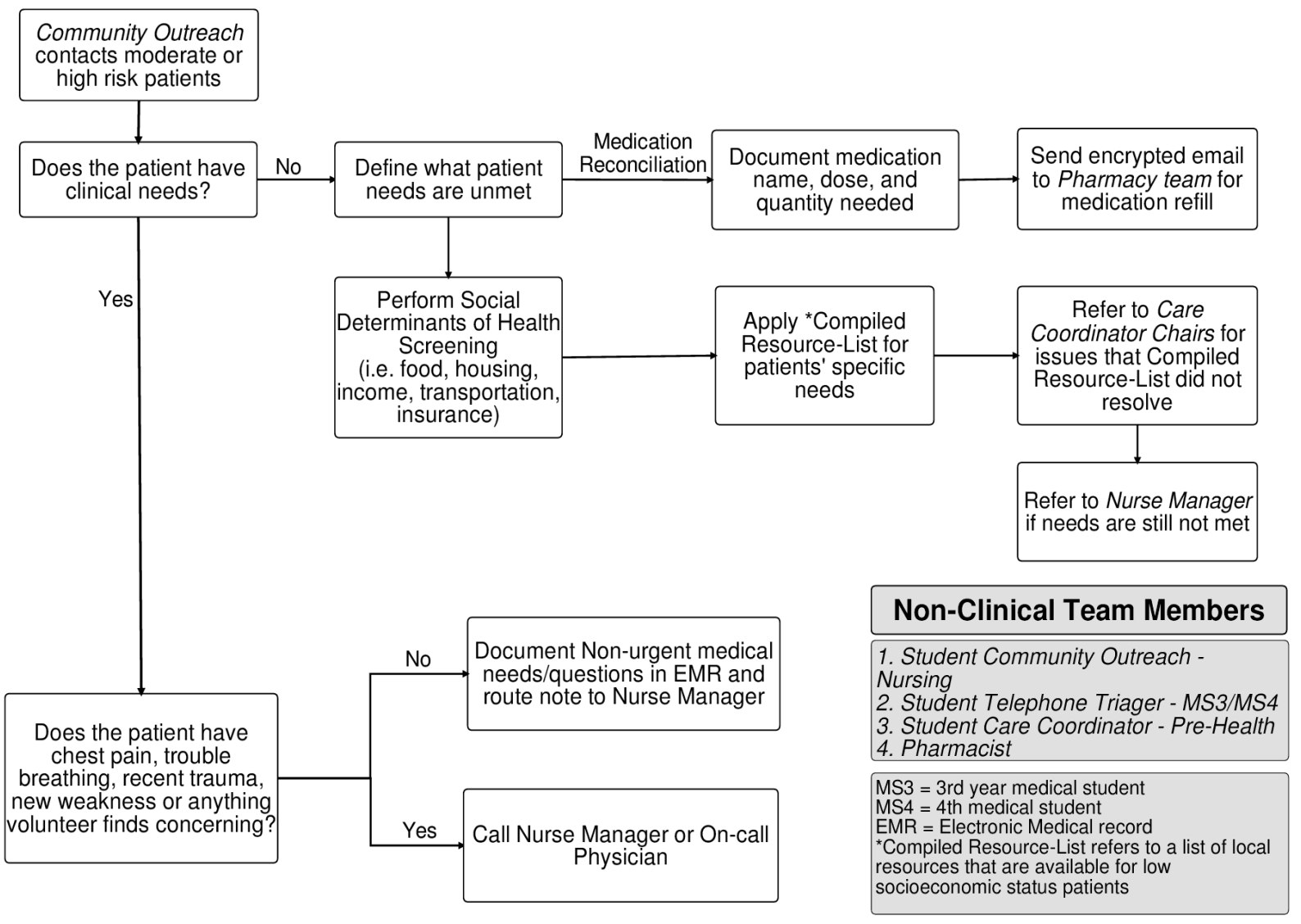

Table 1: DAWN Clinic Visit Data and No-Show Rates, January 2020 to July 2020

\begin{tabular}{|c|c|c|c|c|c|c|c|}
\hline Conducted Visits by Visit Type & January & February & March & April & May & June & July \\
\hline Medicine, telehealth * & 0 & 0 & 0 & 9 & 19 & 24 & 18 \\
\hline Medicine, in-person † & 65 & 52 & 30 & 3 & 2 & 7 & 20 \\
\hline Pharmacy, telehealth * & 0 & 0 & 0 & 2 & 4 & 13 & 6 \\
\hline Pharmacy, in-person $\ddagger$ & 13 & 16 & 4 & 0 & 0 & 0 & 0 \\
\hline Total visits, telehealth * & 0 & 0 & 0 & 11 & 23 & 37 & 24 \\
\hline Visits with interpreter, telehealth * & 0 & 0 & 0 & 8 & 16 & 30 & 15 \\
\hline All visits & 78 & 68 & 34 & 14 & 25 & 44 & 44 \\
\hline \multicolumn{8}{|l|}{ No-Show Rates by Visit Type } \\
\hline Medicine, telehealth & $0 \%$ & $0 \%$ & $0 \%$ & $29 \%$ & $22 \%$ & $9 \%$ & $5 \%$ \\
\hline Pharmacy, telehealth & $0 \%$ & $0 \%$ & $0 \%$ & $50 \%$ & $20 \%$ & $24 \%$ & $14 \%$ \\
\hline In-person visits & \multirow{2}{*}{$28 \%$} & \multirow{2}{*}{$25 \%$} & \multirow{2}{*}{$6 \%$} & $50 \%$ & $50 \%$ & $30 \%$ & $9 \%$ \\
\hline All visits & & & & $33 \%$ & $22 \%$ & $14 \%$ & $6 \%$ \\
\hline
\end{tabular}

Abbreviation: DAWN, Dedicated to Aurora's Wellness and Needs

* Telehealth service unavailable prior to April 2020

† Preceptor-only visits starting March 2020

$\ddagger$ No in-person services after telehealth was established 
Table 2: Telehealth Visit Chief Complaints Data, April 2020 to July 2020

\begin{tabular}{|c|c|}
\hline Acute Concerns & $\begin{array}{l}\text { Total Visits (Total Converting to In- } \\
\text { Person Visit for Examination) }\end{array}$ \\
\hline Hospital discharge follow-up (general) & $14(3) *$ \\
\hline Hospital discharge follow-up (COVID) & $8(3) \dagger$ \\
\hline Chest pain & $4(2)$ \\
\hline Rash & $3(1)$ \\
\hline Shortness of breath & $2(2)$ \\
\hline Swelling & $2(1)$ \\
\hline Musculoskeletal pain/injury & 2 \\
\hline Syncope & 1 \\
\hline Urinary tract infection & 1 \\
\hline Eye problem & 1 \\
\hline \multicolumn{2}{|l|}{ Chronic Concerns } \\
\hline Hypertension & $43(8) \ddagger$ \\
\hline Diabetes care & 28 \\
\hline Hyperlipidemia & 11 \\
\hline Coronary artery disease & 7 \\
\hline Musculoskeletal pain & 7 \\
\hline Hypothyroidism & 5 \\
\hline Abdominal pain (eg, constipation, heartburn) & 5 \\
\hline Headache & 5 \\
\hline Lower urinary tract symptoms (noninfectious) & 5 \\
\hline Neurologic disorder & 3 \\
\hline Chronic kidney disease & 3 \\
\hline Dizziness & $3(1)$ \\
\hline Mental health (eg, anxiety, depression) & 2 \\
\hline Dysfunctional uterine bleeding & 2 \\
\hline Fatigue & $2(1)$ \\
\hline Acne & 2 \\
\hline Obesity & 2 \\
\hline Heart failure & 1 \\
\hline Insomnia & 1 \\
\hline
\end{tabular}

${ }^{*}$ All in-person visits required for volume examination

$\dagger$ All in-person visits required for oxygen titration

$\ddagger$ All in-person visits for in-clinic blood pressure measurement due to no home blood pressure cuff 


\section{Corresponding Author}

Kari Mader, MD, MPH

12631 E. 17th Ave, Rm 3119, Aurora, CO 80045. 303-724-9707.

kari.mader@cuanschutz.edu

\section{Author Affiliations}

Ryan Cao Vinh Phan - University of Colorado School of Medicine, Aurora, CO

Dung Van Le - University of Colorado School of Medicine, Aurora, $\mathrm{CO}$ I and Dedicated to Aurora's Wellness and Needs (DAWN), Aurora, CO

Alexander Nguyen - University of Colorado School of Medicine, Aurora, CO I and Dedicated to Aurora's Wellness and Needs (DAWN), Aurora, CO.

Kari Mader, MD, MPH - Dedicated to Aurora's Wellness and Needs (DAWN), Aurora, CO I and Department of Family Medicine, University of Colorado School of Medicine, Aurora, $\mathrm{CO}$.

\section{References}

1. Courtemanche C, Garuccio J, Le A, Pinkston J, Yelowitz A. Strong social distancing measures in the United States reduced the COVID-19 growth rate. Health Aff (Millwood). 2020:101377hlthaff202000608. doi:10.1377/hlthaff.2020.00608. [Epub ahead of print].

2. Raifman MA, Raifman JR. Disparities in the population at risk of severe illness from COVID-19 by race/ethnicity and income. Am J Prev Med. 2020;59(1):137-139; Epub ahead of print. doi:10.1016/j.amepre.2020.04.003

3. Smith S, Thomas R III, Cruz M, Griggs R, Moscato B, Ferrara A. Presence and characteristics of student-run free clinics in medical schools. JAMA. 2014;312(22):2407-2410. doi:10.1001/jama.2014.16066

4. Association of American Medical Colleges. Medical School Graduation Questionnaire. Washington, DC: AAMC; 2019. https://www.aamc.org/system/files/2019-08/2019-gq-all-schools-summary-report.pdf. Accessed September 14, 2020.

5. Waseh S, Dicker AP. Telemedicine training in undergraduate medical education: mixed-methods review. JMIR Med Educ. 2019;5(1):e12515. doi:10.2196/12515

6. van Veen T, Binz S, Muminovic M, et al. Potential of mobile health technology to reduce health disparities in underserved communities. West J Emerg Med. 2019;20(5):799-802. doi:10.5811/westjem.2019.6.41911

7. Bramstedt KA, Prang M, Dave S, Shin PN, Savy A, Fatica RA. Telemedicine as an ethics teaching tool for medical students within the nephrology curriculum. Prog Transplant. 2014;24(3):294-297. doi:10.7182/pit2014289

8. Goetter EM, Blackburn AM, Bui E, Laifer LM, Simon N. Veterans' prospective attitudes about mental health treatment using telehealth. J Psychosoc Nurs Ment Health Serv. 2019;57(9):38-43. doi:10.3928/02793695-20190531-02

9. Afshari M, Witek NP, Galifianakis NB. Education Research: an experiential outpatient teleneurology curriculum for residents. Neurology. 2019;93(4):170-175. doi:10.1212/WNL.0000000000007848

10. Holekamp NM. Moving from clinic to home: what the future holds for ophthalmic telemedicine. Am J Ophthalmol. 2018;187:xxviii-xxxv. doi:10.1016/j.ajo.2017.11.003

11. Molleda L, Bahamon M, St George SM, et al. Clinic personnel, facilitator, and parent perspectives of eHealth Familias Unidas in Primary Care. J Pediatr Health Care. 2017;31(3):350-361. doi:10.1016/j.pedhc.2016.11.001

12. Kolltveit BH, Gjengedal E, Graue $M$, Iversen MM, Thorne S, Kirkevold M. Conditions for success in introducing telemedicine in diabetes foot care: a qualitative inquiry. BMC Nurs. 2017;16(1):2. doi:10.1186/s12912-017-0201-y

13. Zoom Video. https://zoom.us/. Published 2020. Accessed September 4, 2020.

14. Phan RC, Le DV, Nguyen A, Mader K. Student-run free clinics telehealth platform solutions. 2020. STFM 
Resource Library. https://resourcelibrary.stfm.org/viewdocument/student-run-free-clinic-telehealth-1.

Accessed September 14, 2020.

15. Phan RC, Le DV, Nguyen A, Mader K. Zoom meeting instructions. 2020. STFM Resource Library. https://resourcelibrary.stfm.org/viewdocument/zoom-meeting-instructions. Accessed September 14, 2020.

16. Phan RC, Le DV, Nguyen A, Mader K. DAWN Student-Run Free Clinic Telehealth Student Training Guide. 2020. STFM Resource Library. https://resourcelibrary.stfm.org/viewdocument/dawn-student-run-free-clinic-telehe. Accessed September 14, 2020.

Copyright $\odot 2020$ by the Society of Teachers of Family Medicine 